Strauss, B. S. (1958). J. gen. Microbiol. 18, 658-669

\title{
Gell Death and 'Unbalanced Growth' in Neurospora
}

\author{
By B. S. STRAUSS
}

Department of Zoology, Syracuse University, Syracuse 10, New York, U.S.A.

SUMMARY: Mutant spores of Neurospora crassa incubated on minimal medium showed two main types of behaviour. One mutant type survived incubation for a week on minimal medium and then responded to the addition of growth substance. The second mutant type died after incubation for one or two days. Strains which died were characterized by an ability to germinate in minimal medium and to begin immediately synthetic processes such as the synthesis of protein and nucleic acid. Addition of ethionine to minimal medium prolonged the survival of strains which committed 'suicide'. Non-germinated Neurospora conidia withstood freezing and storage at $-10^{\circ}$ for extended periods, whereas conidia which had been incubated in growth medium died, indicating a difference between dormant and synthetically active spores in their response to the environment. The suicide phenomenon is reminiscent of the cell death due to 'unbalanced growth' reported for the thyminerequiring mutant of Escherichia coli. In this case, however, cells requiring substances other than nucleic acid constituents display the effect.

Mutant strains of the ascomycete Neurospora crassa can be divided into two major types when conidiospores are incubated in a minimal medium. The first type will survive for relatively long periods on an agar plate containing minimal medium and will then respond to the addition of its growth requirement by the production of visible colonies. The second type dies after a short incubation period; i.e. does not respond to addition of growth factor. This behaviour was first observed by Stevens \& Mylroie (1953) during studies on the validity of the 'back-mutation' test in Neurospora, but as yet there have been no studies of the cause of the phenomenon nor on the difference between the two classes of mutants. A very similar phenomenon was described by Fries (1948) in Ophiostoma and by Macdonald \& Pontecorvo (1953) in Aspergillus nidulans. Macdonald \& Pontecorvo showed that spores of a biotin-requiring mutant died off rapidly in a biotin-deficient medium. The very interesting observation was made that in certain cases the introduction of a second mutation drastically increased survival in minimal medium, e.g. with biotin- and adenine-requiring or with biotin- and sulphite-requiring strains. Cohen \& Barner (1954) described a thymine-requiring mutant of Escherichia coli which committed 'suicide' when incubated in a medium devoid of thymine. These authors attributed death of the mutant to unbalanced growth since in the absence of thymine quantities of ribonucleic acid (RNA) and protein were formed but deoxyribonucleic acid (DNA) was not. The organisms swelled and increased in mass but did not divide. Eventually, if allowed to continue to synthesize in a medium devoid of thymine, mutant organisms lost their ability to respond to thymine upon its addition. The cause of 'death' was supposedly due to imbalance in the ratio: (RNA + protein): DNA. 
It is evident that organisms which have lost the ability to synthesize single essential constituents, i.e. biochemical mutants, must perforce carry out unbalanced synthesis when they are incubated in a medium devoid of their growth requirement. Initially, at least, all the synthetic processes which do not involve the missing constituent may proceed, whereas those involving the requirement will not. This condition is, formally at least, one of imbalance. We have therefore attempted to answer two closely related questions: (1) is the 'suicide' of mutant conidia incubated on minimal medium related to their unbalanced synthesis; (2) why do certain mutants survive for long periods on a growth medium lacking only one constituent? As as result of these investigations we are convinced that the suicide effect is due to the initiation of synthetic processes in certain mutant types. Secondly, it appears that starvation death only occurs in spores that have been interrupted in synthetic processes which they have already started. Those mutant spores which survive on minimal medium for long periods do not break the (synthetically) dormant state of the conidiospore and are therefore not susceptible to starvation death.

\section{METHODS}

In most of the previous investigations with Neurospora, conidia were collected from cultures grown on solid medium after growth periods ranging from 4 to 8 days (Strauss, 1956). Spores are formed throughout the growth period and what is actually obtained is a population of spores of different ages. In order to compare the behaviour of spores of different strains we developed a method for the production of spores differing in their ages (from time of formation) by not more than a few hours. This method is based on a method of Zalokar (1954) for the study of carotenoid formation in Neurospora. Mycelial pads are obtained by permitting growth to occur in a $250 \mathrm{ml}$. Erlenmeyer flask for 3 days at $30^{\circ}$ in $50 \mathrm{ml}$. minimal medium (Beadle \& Tatum, 1945) containing $\mathbf{0 . 2 3} \%(\mathrm{w} / \mathrm{v})$ sodium acetate (tri-hydrate) + two drops of ' Tween 80 ' to inhibit the formation of aerial hyphae. At the conclusion of the growth period the pads are harvested on a Buchner funnel, washed thoroughly with sterile distilled water and transferred to Petri dishes containing filter paper. The semi-dry pads are then soaked by the addition of $4 \mathrm{ml} .0 \cdot 1 \mathrm{M}$-phosphate buffer (pH 6.0) and allowed to incubate at $25^{\circ}$. There is no spore formation until after 12-13 hr. (Fig. 1), after which formation of conidia begins and continues to a maximum reached after 15-16 hr. Conidiation of the mutants used (with the exception of the at suc strain) occurs as well in the absence of growth factor as in its presence. It is likely, however, that new protein synthesis is required for conidiation and that the mutants obtain their growth factor from endogenous sources. Ethionine inhibits conidiation and this inhibition is overcome in a competitive fashion by methionine (Table 1). Ammonium ion inhibits conidiation by the at suc strain and this inhibition is overcome by succinate as is ammonium inhibition of the growth process (Strauss, 1956). Inhibition by ethionine is effective only when the ethionine is added within the first $9 \mathrm{hr}$. of incubation. Since the conidia are formed on aerial hyphae 
we suspect that the 'latent period' before conidiation represents the time required for the aerial hyphae to form. The growth process in this conidiation system might well be the production of these hyphae.

\section{Table 1. Interaction of DL-ethionine and L-methionine on conidiation in Neurospora crassa}

Conidiation is recorded as the optical density at $625 \mathrm{~m} \mu$. of the conidial suspension obtained by collecting the conidia produced by one pad, filtering through gauze and then making the suspension to $50 \mathrm{ml}$. Incubation for $16 \mathrm{hr}$. at $25^{\circ}$. Additions in $0 \cdot 1 \mathrm{M}$-phosphate buffer $(\mathrm{pH} \mathrm{6 \cdot 0)}$. Total amount of buffer added in all cases $=4.0 \mathrm{ml}$.

\begin{tabular}{|c|c|c|c|c|}
\hline \multirow{2}{*}{$\begin{array}{l}\text { Concentration of } \\
\text { L-methionine } \\
(\mu \mathrm{M})\end{array}$} & \multicolumn{4}{|c|}{ Concentration of DL-ethionine added $(\mu \mathrm{M})$} \\
\hline & $\mathbf{0}$ & $0 \cdot 5$ & $1 \cdot 0$ & $1 \cdot 5$ \\
\hline & \multicolumn{4}{|c|}{ Degree of conidiation } \\
\hline $\mathbf{0}$ & $\begin{array}{l}0 \cdot 28 \\
0 \cdot 32 \\
0 \cdot 35\end{array}$ & $\begin{array}{c}0 \cdot 21 \\
0 \cdot 24 \\
-\end{array}$ & $\begin{array}{c}0 \cdot 08 \\
0 \cdot 17 \\
-\end{array}$ & - \\
\hline 2 & $\begin{array}{l}0.27 \\
0.20\end{array}$ & $\begin{array}{l}0 \cdot 19 \\
0 \cdot 24\end{array}$ & $\begin{array}{l}0.06 \\
0.08\end{array}$ & $\begin{array}{l}0.05 \\
0.07\end{array}$ \\
\hline 5 & $\begin{array}{l}0 \cdot 20 \\
0 \cdot 22\end{array}$ & $\begin{array}{l}0 \cdot 16 \\
0 \cdot 22\end{array}$ & $\begin{array}{l}0 \cdot 19 \\
0 \cdot 18\end{array}$ & $\begin{array}{l}0 \cdot 15 \\
0 \cdot 09\end{array}$ \\
\hline 10 & $\begin{array}{l}0 \cdot 18 \\
0 \cdot 14\end{array}$ & $\begin{array}{l}0 \cdot 10 \\
0 \cdot 205\end{array}$ & $\begin{array}{l}0.23 \\
0.28\end{array}$ & $\begin{array}{l}0.23 \\
0.24\end{array}$ \\
\hline
\end{tabular}

In the following experiments conidia were prepared by the method indicated. Mutants were grown by the addition of the appropriate growth factor to minimal medium with acetate and 'Tween $80^{\text {'. }}$. Washed mycelial pads were then allowed to incubate in Petri dishes for 17-18 hr. The conidia were collected by washing the surface of the mycelial pad with water and filtering through gauze to remove mycelial fragments. After collection the conidia were washed twice with sterile water by centrifugation. Incubation of conidia in our experiments was generally at $30^{\circ}$ except where otherwise indicated. When incubation was in liquid medium, a digestion tube containing a suspension of conidia ( $35 \mathrm{ml}$.) was immersed in a water bath and kept agitated and aerated by the passage of a current of air.

In the course of these experiments we attempted to measure viability, germination, nuclear division, protein synthesis, total nucleic acid and oxygen consumption. Viability is defined in these experiments as the ability of a conidium to form a visible colony when plated on an agar medium suitably supplemented with growth factor and containing $1 \%(\mathrm{w} / \mathrm{v})$ sorbose $+0 \cdot 1 \%$ (w/v) sucrose (Tatum, Barratt \& Cutter, 1949). Germination is reported as percentage conidiospores with a definite germ tube emerging from the spore. Nuclear division was determined by comparison of the average nuclear count of conidia before and after varying periods of incubation. A modification of the method of Huebschman (1952) described by Zalokar (personal communication) was used. In this method 'fixed' conidia are stained with azure $\mathbf{A}$ after 15 min. hydrolysis with $\mathrm{N}-\mathrm{HCl}$ at $55^{\circ}$. After two sulphite washes the conidial suspension is dehydrated by passing through ethanol and mounted. The whole 
staining procedure is carried out in a centrifuge tube, the conidial suspension being placed on a slide only after the final dehydration. While the Feulgen stain has been successfully used we find, in agreement with Zalokar, that the nuclei stain more clearly with azure A. Total protein synthesis was determined as the amount of ${ }^{35} \mathrm{SO}_{4}$ incorporated into the fraction of conidia remaining after extraction with cold $10 \%(\mathrm{w} / \mathrm{v})$ trichloroacetic acid, $80 \%(\mathrm{v} / \mathrm{v})$ ethanol in water, a mixture of ethanol ( 3 vol. $)+$ diethyl ether $(1$ vol. $)\left(4\right.$ times at $\left.60^{\circ}\right)$ and $0.5 \mathrm{~N}$-perchloric acid $\left(4\right.$ times at $\left.70^{\circ}\right)$. Chromatography of this residual fraction after hydrolysis with $6 \mathrm{~N}-\mathrm{HCl}$ indicated that sulphur was present only as cyst(e)ine and methionine. Total nucleic acid was determined on the perchloric acid extract mentioned above. Nucleic acid was taken as proportional to the optical density of the extract at $260 \mathrm{~m} \mu$. minus the optical density at $290 \mathrm{~m} \mu$., as measured in the Beckman spectrophotometer. The absorption spectrum of the perchloric acid extract indicated a peak at about $260 \mathrm{~m} \mu$. Oxygen consumption was determined by standard manometric techniques.

Organisms. The following strains of Neurospora crassa were used in these investigations. Strain $74 \mathrm{~A}$, a wild-type, and the following amino acidrequiring strains: 584 (methionine), 684 (leucine), 782 (lysine) and 830 (arginine), all obtained from Dr Adrian Srb (Cornell University). The four mutants were derived from the $\mathbf{7 4} \mathrm{A}$ wild-type. Strain 37401, an inositol-requiring strain, and strain $\mathbf{3 7 8 0 3}$, a vitamin $\mathrm{B}_{\mathbf{6}}$-requiring strain, were obtained from Mrs Mary Mitchell (California Institute of Technology). Strains $a d 1$ and $a d$ F 12, both adenine-requiring, were obtained from Dr Norman Giles (Yale University). The at suc strain is the modified succinate-requiring strain previously described (Strauss, 1956). The growth-response curves for four of these strains are given in Fig. 2. The strains used cover a range of growth requirements of about a thousandfold; the requirement of the vitamin $B_{6}$-requiring strain being satisfied by about $2 \mu \mathrm{g}$. $/ 20 \mathrm{ml}$., whereas the amino acid-requiring strains require, typically, about $2 \mathrm{mg}$. $/ 20 \mathrm{ml}$. medium. Growth has been determined as the dry weight of mycelium produced after 3 days by an inoculum of spores into $20 \mathrm{ml}$. medium contained in a $125 \mathrm{ml}$. Erlenmeyer flask and incubated at $25^{\circ}$.

\section{RESULTS}

The inositol-requiring, vitamin $\mathrm{B}_{6}$-requiring and succinate-requiring (at suc) strains started to die rapidly after 1-2 days of incubation on minimal medium (Fig. 3); the leucine-, lysine- and methionine-requiring mutants showed only slight changes in viability even after incubation for 7 days, while the adenineand arginine-requiring mutants were intermediate. The fraction of these latter two strains which survived incubation for 7 days was $c .0 \cdot 3$. In these studies mutant conidia were spread on minimal sorbose plates and then, after the desired time interval, a sufficient quantity of growth factor was injected under the surface of the agar by a hypodermic syringe and allowed to diffuse up through the agar. When conidia of the inositol-requiring strain, 37401, were incubated overnight with shaking in liquid minimal medium they lost their 
ability to take up oxygen even when inositol was added (Table 2) and even though the incubated material had increased in dry weight as compared with non-incubated controls. At the conclusion of the incubation period the inactivated inositol-requiring conidia formed small clumps.

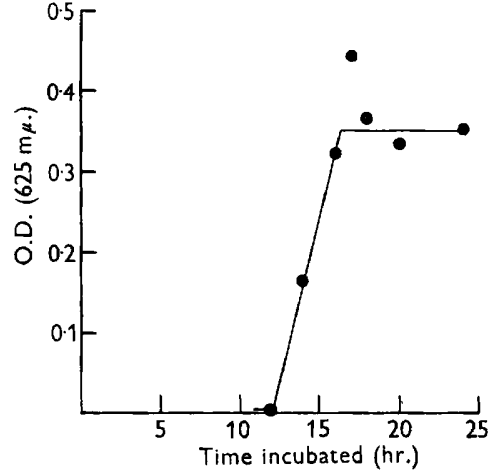

Fig. 1

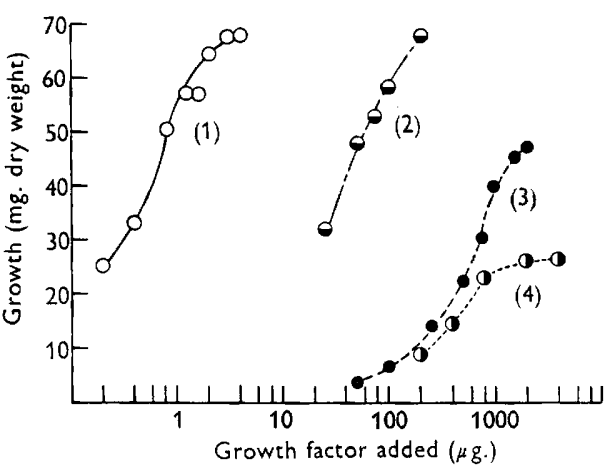

Fig. 2

Fig. 1. Conidiation as a function of incubation time. Conidiation is recorded as the optical density at $625 \mathrm{~m} \mu$. of the conidial suspension obtained by collecting the conidia produced by one pad, filtering through gauze to remove mycelial fragments and then making the suspension to $35 \mathrm{ml}$. Incubation was at $25^{\circ}$ with $4 \mathrm{ml}$. of $0.1 \mathrm{M}$-phosphate buffer (pH 6.0). Values are the average of duplicates.

Fig. 2. Response of mutants of Neurospora crassa to their required growth factor. The amount of growth factor indicated was added to $20 \mathrm{ml}$. minimal medium. Growth is recorded as the $\mathrm{mg}$. dry weight mycelium produced after incubation for 3 days at $25^{\circ}$ in static culture. Curve (1): mutant 37803, vitamin $B_{6}$-requiring; curve (2): mutant 37401, inositol-requiring ; curve (3): mutant $a d \mathrm{~F} 12$, adenine-requiring; curve (4) : mutant 684, leucine-requiring.

Table 2. Loss of the ability of the inositol-requiring mutant (37401) of

Neurospora crassa to respire after incubation in minimal medium

Equal volumes of a conidial supension of mutant 37401 were added to a series of flasks containing $50 \mathrm{ml}$. minimal medium. Refrigerated set kept $24 \mathrm{hr}$. standing in cold. Incubated-shaken $24 \mathrm{hr}$. at $28^{\circ}$. Conidia were collected and washed twice, and one set was used for dry weight. Material from the other flasks was pooled and made to $10 \mathrm{ml}$. with minimal medium. $2 \mathrm{ml}$. of the resulting suspension was added to manometers and the oxygen consumption was determined at $30^{\circ}$.

\begin{tabular}{|c|c|c|c|}
\hline \multirow[b]{2}{*}{ Condition } & \multirow[b]{2}{*}{$\begin{array}{l}\text { Dry wt. after } \\
\text { experimental } \\
\text { period (mg.) }\end{array}$} & \multicolumn{2}{|c|}{ Oxygen uptake ( 1 hr.; $\mu \mathrm{l})}$. \\
\hline & & $\begin{array}{l}\text { Minimal } \\
\text { medium }\end{array}$ & $\underset{\text { inositol }}{\text { Minimal }}$ \\
\hline Refrigerated & $10 \cdot 1$ & $\begin{array}{l}171 \\
179\end{array}$ & $\begin{array}{l}163 \\
199\end{array}$ \\
\hline Incubated at $28^{\circ}$ & $\mathbf{2 3} \cdot \mathbf{4}$ & $\begin{array}{l}7 \\
0\end{array}$ & $\begin{array}{l}15 \\
10\end{array}$ \\
\hline
\end{tabular}

The inositol- and vitamin $\mathbf{B}_{6}$-requiring strains germinated readily in minimal medium (Fig. 4), the amino acid-requiring mutants did not germinate at all after aeration for $6 \mathrm{hr}$. in minimal medium, while the adenine-requiring strains showed very slight germination. We used a conservative estimation in these experiments; a spore was scored as germinated only when an unambiguous 
outgrowth was seen. In most of the following experiments we arbitrarily used the inositol-requiring strain as typical of those strains which kill themselves, while the leucine-requiring strain was selected as typical of strains which survived.

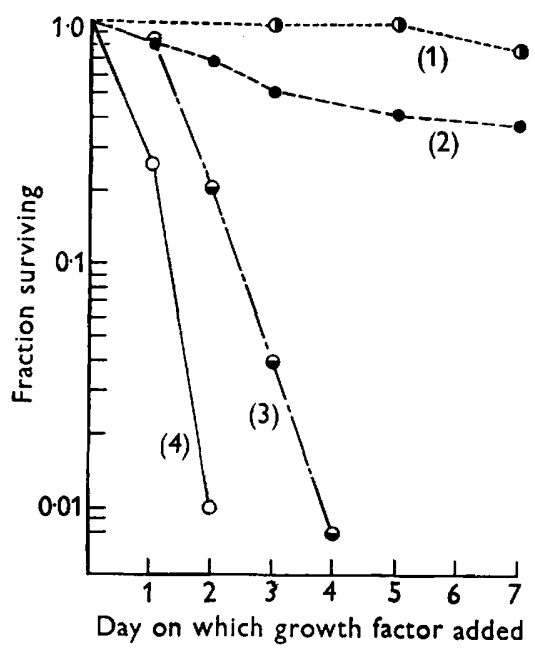

Fig. 3

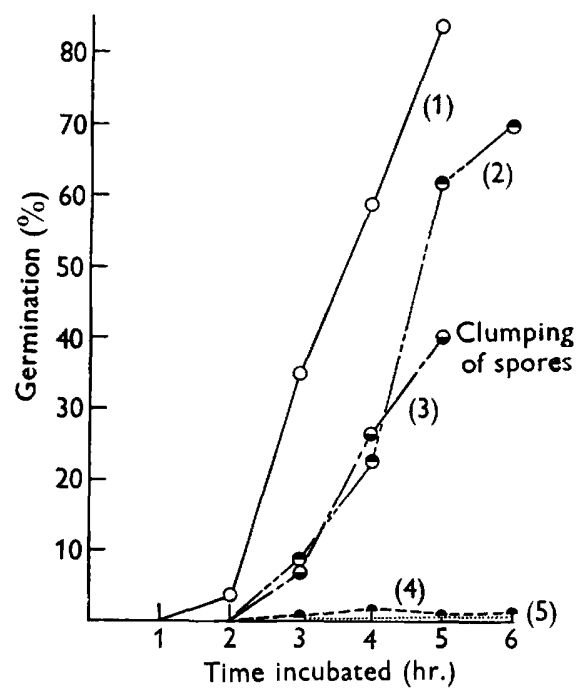

Fig. 4

Fig. 3. Survival of mutants of Neurospora crassa incubated on minimal medium. Growth factor was injected under the surface of the agar after incubation at $25^{\circ}$ for the time indicated. The plates were then incubated an additional $3-4$ days. Curve (1): leucinerequiring; curve (2): adenine-requiring; curve (3): inositol-requiring; curve (4): vita$\min \mathbf{B}_{6}$-requiring.

Fig. 4. Germination of mutant and wild-type strains of Neurospora crassa. Conidia were incubated with aeration in $35 \mathrm{ml}$. minimal medium at $30^{\circ}$ for the times indicated. Germination was determined by microscopic count of formalin-fixed material; at least 500 conidia were scored for each point. Curve (1): vitamin $B_{6}$-requiring; curve (2): wild-type; curve (3): inositol-requiring; curve (4): adenine-requiring; curve (5): leucine-requiring.

Germination represents true synthesis on the part of the conidia since the formation of an outgrowth was invariably preceded by protein and nucleic acid synthesis (Figs. 5, 6). We have not, as yet, been able to measure the DNA content of Neurospora conidia so that we cannot compare RNA:DNA ratios as was done by Cohen $\&$ Barner (1954). It has been possible to show an increase in the average number of nuclei/conidium on incubation of both wildtype and the inositol-requiring strain in minimal medium by the azure $A$ technique and by the increase in the number of Feulgen-staining bodies (Table 3). It is therefore quite likely that the increase in total nucleic acid includes an increase in DNA.

The Neurospora conidium is multinucleate; a population of conidia of strain $74 \mathrm{~A}$ has a distribution of nuclei/conidium with an average of $2 \cdot 6$. This average may differ from strain to strain and is determined partly by the medium on which the conidiating mycelium is grown (Huebschman, 1952). The average number of nuclei/conidium increases on incubation in minimal 
medium and has increased to an average of 3.5 after incubation for $2 \mathrm{hr}$. After incubation for $2 \mathrm{hr}$. no germination can be discerned. It therefore seems that nuclear division precedes germination in Neurospora.

Table 3. Distribution of nuclei in Neurospora conidia

\begin{tabular}{|c|c|c|c|c|c|}
\hline \multirow[b]{2}{*}{ Strain of $N$, crassa } & & \multicolumn{4}{|c|}{ Duration of incubation (hr.) } \\
\hline & & $\mathbf{0}$ & 1 & $\mathbf{2}$ & 4 \\
\hline Wild-type (74A) & $\begin{array}{l}\text { Conidia counted } \\
\text { Mean : nuclei/conidium } \\
\text { Mode: nuclei/conidium }\end{array}$ & $\begin{array}{l}454 \\
2 \cdot 61 \\
2\end{array}$ & $\begin{array}{c}556 \\
2 \cdot 76 \\
2\end{array}$ & $\begin{array}{c}566 \\
3 \cdot 47 \\
3\end{array}$ & $\begin{array}{l}526 \\
\quad 5 \cdot 42 \\
5\end{array}$ \\
\hline Inositol-requiring (37401) & $\begin{array}{l}\text { Conidia counted } \\
\text { Mean: nuclei/conidium } \\
\text { Mode: nuclei/conidium }\end{array}$ & $\begin{array}{l}580 \\
2 \cdot 36 \\
2\end{array}$ & $\begin{array}{l}592 \\
2 \cdot 79 \\
2\end{array}$ & $\begin{array}{c}669 \\
3 \cdot 29 \\
3\end{array}$ & $\begin{array}{l}481 \\
4.97 \\
5\end{array}$ \\
\hline Leucine-requiring (684) & $\begin{array}{l}\text { Conidia counted } \\
\text { Mean: nuclei/conidium } \\
\text { Mode : nuclei/conidium }\end{array}$ & $\begin{array}{l}620 \\
2 \cdot 38 \\
2\end{array}$ & $\begin{array}{l}385 \\
2 \cdot 56 \\
2\end{array}$ & $\begin{array}{c}419 \\
2 \cdot 48 \\
2\end{array}$ & $\begin{array}{l}461 \\
2 \cdot 55 \\
2\end{array}$ \\
\hline
\end{tabular}

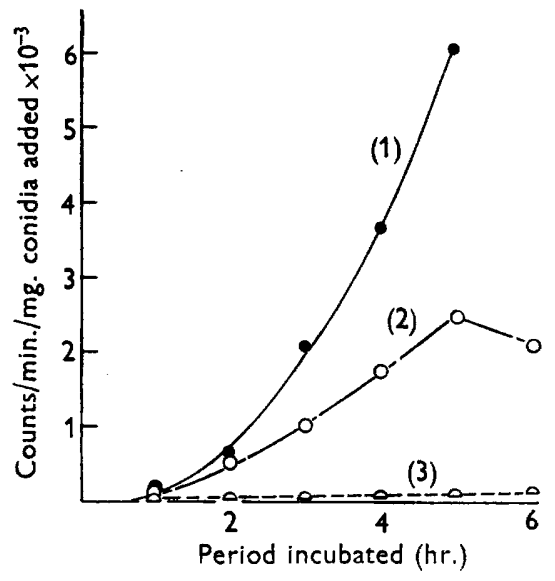

Fig. 5

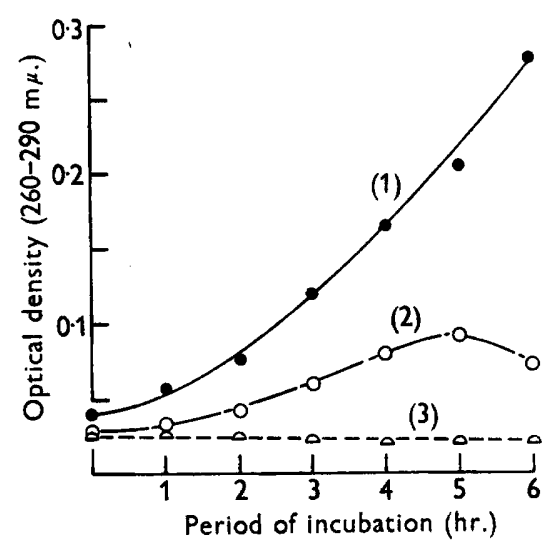

Fig. 6

Fig. 5. Protein synthesis by conidia of Neurospora crassa. Conidia were incubated as before

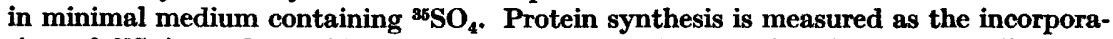
tion of ${ }^{a s} S$ into the residual fraction (see text for details). Curve (1): wild-type; curve (2): inositol-requiring; curve (3): leucine-requiring.

Fig. 6. Total nucleic acid synthesis by conidia of Neurospora crassa. Conidia were incubated in minimal medium as before. Nucleic acid synthesis was measured as the difference in optical density (260-290 $\mathrm{m} \mu$.) of the material extracted with perchloric acid after preliminary cold acid and ether + ethanol extraction (see text for details). Curve (1): wild-type; curve (2): inositol-requiring; curve (3): leucine-requiring.

There was no major difference in the rate at which conidia of the leucinerequiring mutant, which did not commit suicide, took up oxygen when suspended in buffer as compared to wild-type conidia or to conidia of the inositolrequiring strain which did commit suicide (Table 4). Furthermore, conidia of both mutant strains were able to oxidize sucrose both in buffer and in minimal medium at a rate comparable to that of the wild-type. It is difficult to decide whether the increased rate of oxygen uptake in minimal medium compared 
to sucrose buffer shown by both wild-type and inositol-requiring strains during the first hour of incubation was significant, but at any rate this increase was not shown by conidia of the leucine-requiring strain. It is evident that conidia of both the wild-type and mutant strains oxidized carbohydrate at essentially the same rate. Neither of the two mutant strains can be considered to produce spores which are 'dormant' compared to the wild-type as far as their ability to degrade carbohydrate is concerned.

\section{Table 4. Oxygen uptake by wild-type and mutant conidia of Neurospora crassa}

Recorded $\mathrm{O}_{2}$ consumption at $30^{\circ}$ per conidium during the first hour after addition. Manometer contents : main compartment-2.0 ml. conidial suspension in $0.1 \mathrm{M}$-phosphate buffer (pH 6.0); side arm-(a) $0.5 \mathrm{ml}$. buffer, (b) $0.5 \mathrm{ml} .10 \%$ sucrose, (c) $0.5 \mathrm{ml}$. of minimal medium at fourfold concentration.

\begin{tabular}{|c|c|c|c|c|}
\hline \multirow{2}{*}{ Strain of $N$. crassa } & \multicolumn{4}{|c|}{ Addition } \\
\hline & $\begin{array}{l}\text { No of } \\
\text { conidia } \\
\text { added }\end{array}$ & Buffer & Sucrose & $\begin{array}{l}\text { Minimal } \\
\text { medium }\end{array}$ \\
\hline & & \multicolumn{3}{|c|}{ Oxygen consumption $\left(\mu \mathrm{l} . \mathrm{O}_{2} / \mathrm{hr} . /\right.$ conidium $)$} \\
\hline Wild-type (74A) & $1.7 \times 10^{8}$ & $3.7 \times 10^{-7}$ & $\begin{array}{l}24.1 \times 10^{-7} \\
22.5 \times 10^{-7}\end{array}$ & $27 \cdot 4 \times 10^{-7}$ \\
\hline Inositol-requiring (37401) & $2 \cdot 12 \times 10^{8}$ & $4 \cdot 2 \times 10^{-7}$ & $\begin{array}{l}16.5 \times 10^{-7} \\
15.8 \times 10^{-7}\end{array}$ & $\begin{array}{l}20.0 \times 10^{-7} \\
20.0 \times 10^{-7}\end{array}$ \\
\hline Leucine-requiring (684) & $3.06 \times 10^{8}$ & $4 \cdot 4 \times 10^{-7}$ & $\begin{array}{l}22.4 \times 10^{-7} \\
22.8 \times 10^{-7}\end{array}$ & $\begin{array}{l}21.9 \times 10^{-7} \\
23.2 \times 10^{-7}\end{array}$ \\
\hline
\end{tabular}

We attempted without any positive result to determine whether the inositolor vitamin $\mathrm{B}_{6}$-requiring strains incubated in minimal medium produced some soluble product which was either toxic to these strains or to wild-type, or which stimulated germination. Filtrates of minimal medium in which dense suspensions of the inositol- or vitamin $\mathbf{B}_{6}$-requiring mutants had been incubated overnight with shaking did not affect the germination, ability to form colonies on suitable medium or the dry weight attained by either the wild-type or the mutants when such filtrates were added to appropriate media.

Since it seemed possible that the growth of the mutants placed on minimal plates contributed in some way to their death, the effect of an inhibitor of germination on the survival of mutant conidia was determined. Ethionine was selected as the inhibitor because it readily inhibited germination and because its effects could be counteracted by the injection of methionine into the minimal plate containing ethionine at the same time, when growth factor was added. Ethionine had a very marked effect in prolonging survival (Fig. 7) when small amounts were incorporated into minimal medium. At the ethionine concentration used, growth eventually occurred since wild-type conidia plated on medium containing ethionine at this concentration eventually produced colonies. The phenylalanine analogue, DL- $p$-fluorophenylalanine, also prolonged surival of the vitamin $\mathbf{B}_{6}$-requiring mutant in a similar experiment. Lowering the temperature of incubation also increased survival. Neurospora 
conidia retained their viability after being quick frozen and conidia of the inositol-requiring strain kept at $-10^{\circ}$ for 6 days formed colonies on thawing and the addition of growth factor.

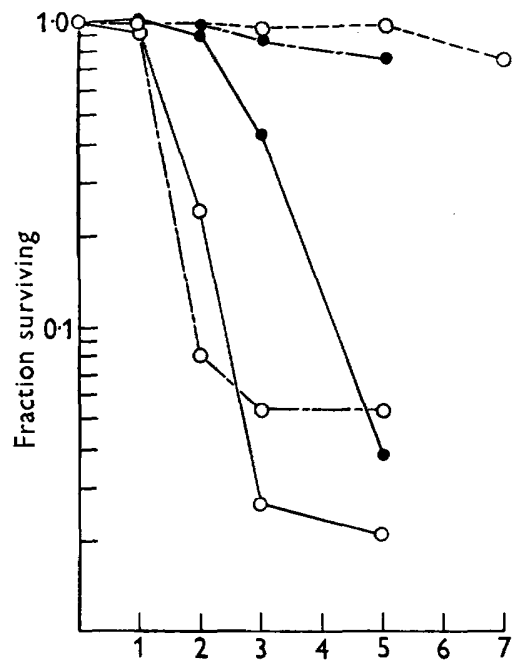

Day on which growth factor added

Fig. 7

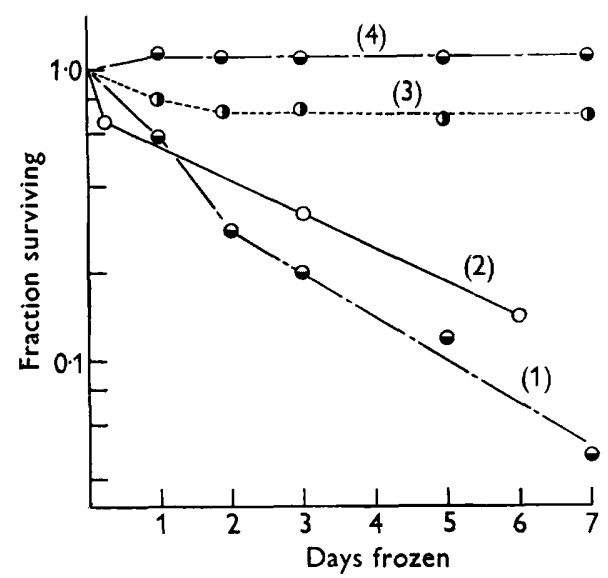

Fig. 8

Fig. 7. Effect of ethionine on the survival of Neurospora crassa mutants incubated on minimal medium. Open circles: cultures incubated on minimal medium. Filled circles : cultures incubated on minimal medium ( $25 \mathrm{ml}$.) containing $0 \cdot 2 \mu$ mole DL-ethionine/ml. Five $\mu$ moles L-methionine were injected along with growth factor at the times indicated. $\longrightarrow$, inositol-requiring; - $-\ldots$, vitamin $\mathbf{B}_{6}$-requiring; $-\ldots-$, , leucine-requiring.

Fig. 8. Effects of storage at $-10^{\circ}$ on the viability of Neurospora crassa conidia incubated for $2 \mathrm{hr}$. in minimal medium before freezing. Viability was determined by colony count after thawing and plating the suspensions. Curve (1): inositol-requiring; curve (2): wild-type; curve (3): leucine-requiring; curve (4): inositol-requiring incubated in buffer.

During the course of these experiments it was noted that the behaviour of conidia after freezing at the temperature of acetone-dry ice mixture and then storing at $-10^{\circ}$ depends on the metabolic condition of the conidia. Wild-type conidia incubated for $2 \mathrm{hr}$. in minimal medium before freezing gradually died on storage at $-10^{\circ}$, in contrast to non-incubated controls. The inositol-requiring mutant mimicked the behaviour of the wild-type (Fig. 8), but conidia of the leucine-requiring mutant remained viable after freezing and storage at $-10^{\circ}$ for the length of the experimental period. Incubation must be in minimal medium since conidia of the inositol-requiring mutant incubated in buffer show no difference in survival after freezing from their non-incubated controls.

\section{DISCUSSION}

If the strains studied are typical then the 'suicide' effect must be related to the start of growth in those strains which kill themselves. The inositol-requiring strain, used as an example of a mutant which commits suicide in 
minimal medium, starts to synthesize protein and nucleic acid, its nuclei divide, and a certain proportion of the spores send out germ tubes. In contrast, the leucine-requiring strain, which does not commit suicide, carries out none of these activities during incubation for $6 \mathrm{hr}$. There is no difference between the ability of conidia of these two strains to oxidize carbohydrate (Table 4) and therefore the differences between them are more likely related to their different synthetic capacities. The effect of ethionine and of fluorophenylalanine in prolonging survival is most easily explained in terms of this hypothesis. We suppose that ethionine prolongs survival by temporarily preventing the initiation of synthetic processes, thereby making conidia of the inositol-requiring and other 'suicidal' mutants similar to those which do not kill themselves off. In essence, the mutants incubated in the presence of growth factor analogue behaved in the same way as the double mutants prepared by Macdonald \& Pontecorvo (1953). With some exceptions these double mutants show increased survival on incubation in minimal medium as compared to the single mutant which dies off most rapidly.

We believe that the data contain clues to the questions $(a)$ why are only actively-synthesizing spores susceptible to death by starvation, and $(b)$ what is the difference between strains which do or do not commit suicide? Assuming that the genetic blocks in these mutants are complete, or almost so, any growth which occurs in minimal medium must be due to material that has been stored in the conidium. A rough idea of the amount of growth that is likely to be obtained might then be obtained from the growth-response curves. Strains that have only a small growth requirement are more likely to have stored sufficient growth substance in their conidia to initiate growth. The strain with the smallest growth requirement (vitamin $\mathbf{B}_{\mathbf{6}}$-requiring) germinates most readily in minimal medium, whereas the strain with the highest growth requirement (leucine-requiring) does not germinate at all during the time period of these experiments (Figs. 2, 4). There is a similar correlation between growth requirement and the suicide effect. The strain with a low growth requirement also kills itself rapidly; the adenine-requiring strain, with an intermediate-high growth requirement, dies only slowly and the strains with the highest quantitative growth requirement (i.e. the amino acid-requiring strains) do not die at an appreciable rate.

This analysis of the relation between growth requirement and the suicide effect is confirmed by the experience of workers using the filtration-selection technique (Woodward, De Zeeuw \& Srb, 1954) for mutant isolation (Drs Srb and Giles, personal communications). This technique is excellent for the isolation of amino acid-requiring mutants, but the yield of vitamin-requiring mutants is low and Dr Giles has not obtained inositol-requiring mutants by this procedure. Since the method requires incubation of mutant spores in minimal medium for long periods, the difficulty in isolating mutants with low growth requirements is easily understood.

The hypothesis requires the supposition that non-germinated conidia differ in some significant way in their response to starvation from conidia which have started to synthesize. While we have no idea as to why this must be so 
the data show that there is a major difference in the response of the organism to its environment in these two different physiological states. Once synthesis has started, conidia are susceptible to death when kept at low temperature; when synthesis has not started, conidia of the same strains are resistant to cold death (Fig. 8). It is not the freezing per se which causes death but rather the storage period, since the strains used show no loss of viability if they are thawed and plated on growth medium immediately after freezing. The cold death phenomenon cannot be explained as due to mechanical effects caused by the absorption of water during the incubation period with the subsequent formation of ice crystals since $(a)$ incubation of the inositol-requiring strain in buffer did not lead to a decreased resistance to cold storage, and $(b)$ incubation of the leucine-requiring mutant in minimal medium did not lead to a decreased resistance even though, as has been shown above (Table 4), the leucine-requiring mutant oxidizes sugar readily in minimal medium. The cold death phenomenon must therefore be related to synthetic metabolism. Interruption of synthetic processes (by cold) once begun is lethal for the wildtype and for the inositol-requiring mutant.

There is one major exception to the correlation between growth initiation and the suicide effect. The succinate-requiring mutant, at suc, dies on minimal medium after incubation for 2 days. It is not possible to detect any significant increase in nucleic acid or protein and there is no germination of this strain, after incubation in minimal medium. We do not feel that this exception refutes the hypothesis. It has previously been reported (Strauss, 1956) that there is an unstable balance between carbohydrate and nitrogen metabolism in the succinate-requiring mutants; ammonium salts inhibit the at suc mutant and lead to the accumulation of keto acids and acetylmethylcarbinol. On minimal medium therefore, the at suc strain is unbalanced in its degradative metabolism in contrast to the other strains. The accumulation of products and the inhibition of the carbohydrate oxidation of this strain in the presence of ammonium salts might reasonably be expected to lead to cell death even in the absence of protein and nucleic acid synthesis.

We therefore feel that the death of mutants of Neurospora when incubated on minimal medium is a case of 'unbalanced growth'. Starvation for a component in Neurospora, is only effective as a cause of death when active synthesis is proceeding. This must mean that death ensues when the reaction pathways which utilize the missing or limiting component cannot 'keep up' with the other reaction pathways and this in turn may reasonably be defined as a condition of imbalance. Active synthesis in the absence of the required growth factor is necessary but apparently not sufficient to lead to cell death. A thiamine-requiring mutant of Neurospora germinated in minimal medium but did not die after 7 days' incubation on minimal medium, and a pantothenic acid-requiring mutant germinated but did not start to die off rapidly until after 5 days' incubation on minimal medium. There appears to be something specific about the factors which lead to imbalance and death. This fact was clearly recognized by Macdonald \& Pontecorvo (1953) as a result of their survey of mutant types. 
Assuming that the cause of death in Ophiostoma and in Aspergillus mutants is similar to that in Neurospora, cell death due to 'unbalanced growth' has been recorded in three fungi as well as in Escherichia coli (Cohen \& Barner, 1954). In the case of the fungi at any rate this death is not restricted to cells requiring DNA constituents. Our use of the term 'imbalance' is only intuitive and is as imprecise as the use made of it by Cohen \& Barner (1954). It is quite evident in their case, as in ours, that there is an imbalance but it is far from clear why such an imbalance should lead to cell death.

The author would like to express his gratitude to Dr A. Srb, Dr N. Giles and to Mrs Mary Mitchell for supplying the mutant strains used in this investigation. He would also like to acknowledge the expert technical assistance of Mrs Barbara Wagner. This work was supported under contract AT (30-1) 1138 between Syracuse University and the United States Atomic Energy Commission and by a grant from the National Science Foundation (U.S.).

\section{REFERENCES}

Beadle, G. W. \& Tatum, E. L. (1945). Neurospora II. Methods of producing and detecting mutations concerned with nutritional requirements. Amer. J. Bot. $32,678$.

Cohen, S. S. \& Barner, H. (1954). Studies on unbalanced growth in Escherichia coli. Proc. nat. Acad. Sci., Wash. 40, 885.

Fries, N. (1948). Viability and resistance of spontaneous mutations in Ophiostoma representing different degrees of heterotrophy. Physiol. Plant. 1, 330.

Huebschman, C. (1952). A method for varying the average number of nuclei in the conidia of Neurospora crassa. Mycologia, 44, 599.

Macdonald, K. D. \& Pontecorvo, G. (1953). 'Starvation' technique. Advanc. Genet. 5, 159.

Stevens, C. M. \& Mrlrore, A. (1953). Inhibition effects in back-mutation tests with mutants of Neurospora. Nature, Lond. 171, 179.

Strauss, B. S. (1956). The nature of the lesion in the succinate-requiring mutants of Neurospora crassa: Interaction between carbohydrate and nitrogen metabolism. J. gen. Microbiol. 14, 494.

Tatum, E. L., Barratt, R. W. \& Cutter, V. M., Jr. (1949). Chemical induction of colonial paramorphs in Neurospora and Syncephalastrum. Science. 109, 509.

Woodward, V. W., De Zeeuw, J. R. \& SRB, A. M. (1954). The separation and isolation of particular biochemical mutants of Neurospora by differential germination of conidia, followed by filtration and selective plating. Proc. nat. Acad. Sci., Wash. 40, 192.

Zalokar, M. (1954). Studies on biosynthesis of carotenoids in Neurospora crassa. Arch. Biochem. Biophys. 50, 71. 